\title{
Compliance with Standard Precautions Among Operating Room Nurses at a University Hospital, Egypt
}

\author{
Bishoy Magdy Girgis Awadalla*, Amal Fakhry Garas and Naglaa Fawzy Hanafy \\ Faculty of Nursing, Cairo University, Egypt \\ *Corresponding author: Bishoy Magdy Girgis Awadalla, Faculty of Nursing, Cairo University, Egypt. \\ To Cite This Article: Bishoy Magdy Girgis Awadalla. Compliance with Standard Precautions Among Operating Room Nurses at a University \\ Hospital, Egypt. Am J Biomed Sci \& Res. 2019 - 3(3). AJBSR.MS.ID.000676. DOI: 10.34297/AJBSR.2019.03.000676
}

Received: April 09, 2019 | Published: June 13, 2019

\begin{abstract}
Standard precautions (SPs) have been introduced as means to protect health care workers (HCWs) from exposure to blood borne pathogens (BBPs). Few studies have been conducted to identify the level of compliance with SPs among nurses.
\end{abstract}

Aim: This study determined the level of compliance with SPs among operating room (OR) nurses.

Design: A cross sectional survey was carried out in this study.

Sample: A convenient sample of 37 OR nurses working in the morning shift.

Setting: The sample was recruited from three specialty areas (general, cardiothoracic and orthopedic surgery) at a university hospital, Egypt.

Methodology: A Compliance Assessment Sheet (CAS) consisted of two sections; The first section was used to collect self-reported data on (A) Demographic variables related to the OR observed subject and (B) Background data related to OR nurses' variables; The second section was observation checklist that identified the occurrence of eight SP practices.

Results: The result showed low compliance level with SPs. None of OR nurses in the three specialty areas complied with goggles wear. The highest level of compliance with SPs was among OR nurses in cardiothoracic surgery; while general surgery was the lowest in compliance.

Conclusion: The study concluded that, the levels of compliance among OR nurses under the study were low.

Recommendation: The results can be used to enhance nurses' safety by focusing on areas of non-compliance.

Keywords: Compliance; Standard precautions (SPs); Operating room (OR); Blood borne pathogens (BBPs); Health care workers (HCWs)

\section{Introduction}

Standard precautions have been reported as an effective means of providing protection, both to health care workers (HCWs) and to patients [1,2]; but only when they are systematically and fully implemented [3]. Although SPs are simple to understand and implement, there is a marked reluctance among HCWs to comply with them fully [4].

Standard precautions considered as barriers and work practice controls established to prevent sharp injuries and blood exposure. Despite these precautions, there was limited success in reducing the incidence of sharp injuries due to sub-optional adherence to SPs $[5,6]$. Results of the current study provides information regarding the level of compliance with SPs among OR nurses and help in identifying areas for training and education. It may also be used to evaluate progress in compliance among OR nurses over time.

\section{Methodology}

Aim of the Study: The aim of this study was to determine the level of compliance with SPs among OR nurses at university hospital in Egypt.

Research Questions: To achieve the aim of this study the following research question was formulated: What is the level of compliance with SPs among OR nurses under study?

Setting: At Kaur EL Aini Hospital; General surgery theaters include eight theaters, four in each side; while the cardiothoracic 
surgery is divided into two main theaters. The general and cardiothoracic surgery theaters are located in the third floor. The orthopedic surgery is located in the second floor and includes five main theaters.

Sampling: A convenient sample of 37 OR scrub nurses working in the morning shift in three selected specialty areas (general, cardiothoracic, and orthopedic surgeries) were recruited as a study sample. Nurses with a working experience less than one year were excluded from the study. The entire sample of nurses was (16 in general, 9 in cardiothoracic, and 12 in orthopedic surgeries).

Instruments: A tool was designed by the investigator to collect data pertinent to the study variables. It was developed after extensive literature review and submitted to a panel of three reviewers and experts in medical surgical and OR nursing. Revision of the tool was made based on feedback from reviewers and before conducting a pilot study. The tool (Compliance Assessment Sheet) (CAS) consists of two sections:

I. The first section was used to collect self-reported data on (A) demographic variables related to the OR observed subjects as age, gender, educational qualification, years of working experience in the nursing field; and in the OR, and number of OR nurses in the three surgical specialty areas, in addition to (B) background data related to OR nurses' variables as in-service training courses in infection control (IC), vaccination status against hepatitis $\mathrm{C}$ virus (HBV), OR nurses' awareness regarding IC policies and procedures, number of surgical procedures done in each specialty area, and hours of observation.

II. The second section was the observation checklist that identified the occurrence of eight SP practices. It included a list of compliance with SPs performance criteria of each standard with Yes/No option to designate whether the practice was performed correctly or not; because the importance of compliance to SPs, each practice was counted correct only when all required criteria "sub items" were met.
Practices were observed and recorded by the researcher. Standard Precaution practices were related to: headgear (two items), surgical mask (five items), goggles (two items), OR boots (three items), surgical hand washing (fifteen items), surgical gown (seven items), surgical gloves (ten items) and handling sharp instruments (six items). The observed SPs compliance were quantified as percentage [the denominator (expected) was the total number of potential situations calling for SPs, and the nominator (observed) the actual number of SP items precautionary were implemented correctly. The level of compliance of each SP practice and the total of the eight standards were grouped into low (0\%$79 \%)$, moderate (80\%-99\%) and high (100\%).

Procedure: The research proposal was approved by the ethical committee at the Faculty of Nursing, Cairo University, after which an official permission for conducting the study was obtained from the hospital director as well as the head of the ORs, and nurse supervisors. Measures were taken to protect the subjects' ethical rights. Each potential subject was informed and signed an informed consent clarifying the purpose and the nature of the study. Voluntary participation, confidentiality and anonymity of responses were assured. The researcher developed a schedule in order to observe SP practices in the three OR specialty areas equally and randomly by rotation. Each observation period covered the eight practiced SP measures. Observation started from the beginning of putting on the headgear, and then all the followed standards till the end of the surgical procedure without interrupting the sterility of the surgical field and with good visualization of the practice. Practices were recorded using the observation checklist. Data collection period continued for six months started from April till October 2012. A total of 96 surgical procedures were attended and 768 SP practices were monitored with a total hour 457 hours.

Data Analysis: Upon completion of data collection, the data were coded, tabulated, and analyzed by computer using the "Statistical Package for Social Sciences" (SPSS) 11.5 version. Descriptive statistics such as frequency, percentage, mean, and standard deviation were utilized to analyze data pertinent to the study variables.

Results

\begin{tabular}{|c|c|c|}
\hline Demographic Variables & No. & $\%$ \\
\hline \multicolumn{3}{|l|}{ Age Group (yrs) } \\
\hline $20-$ & 4 & $11 \%$ \\
\hline $25-$ & 3 & $8 \%$ \\
\hline $30-$ & 9 & $24 \%$ \\
\hline $35-$ & 15 & $41 \%$ \\
\hline $40-$ & 5 & $13 \%$ \\
\hline $45-$ & 1 & $3 \%$ \\
\hline Mean \pm SD & \multicolumn{2}{|c|}{$35.08 \pm 6.47$} \\
\hline \multicolumn{3}{|l|}{ Gender } \\
\hline Male & 7 & $19 \%$ \\
\hline Female & 30 & $81 \%$ \\
\hline
\end{tabular}




\begin{tabular}{|c|c|c|}
\hline Educational Qualification & 34 & $91.90 \%$ \\
\hline Diploma Nursing & 3 & $8.10 \%$ \\
\hline Technical Nursing & 3 & $8 \%$ \\
\hline Working Experience in Nursing Field (yrs) & 4 & $11 \%$ \\
\hline $1-$ & 30 & $81 \%$ \\
\hline $5-$ & & \\
\hline $10-$ & 6 & $16 \%$ \\
\hline Working Experience in the OR (yrs) & 5 & $14 \%$ \\
\hline $1-$ & 26 & $70 \%$ \\
\hline $5-$ & & \\
\hline $10-$ & 16 & \\
\hline OR Nurses in Surgical Specialty Areas & 9 & \\
\hline General & 12 & \\
\hline Cardiothoracic & \multicolumn{2}{|c|}{} \\
\hline Orthopedic & \multicolumn{2}{|c|}{} \\
\hline
\end{tabular}

Table 1 shows that OR nurses aged 35 to less than 40 years old had the highest frequency 15/37 (41\%), and those aged more than 45 years old had the lowest frequency $1 / 37(3 \%)$ with a mean of $35.08 \pm 6.47$ years old. There were more females $30 / 37(81 \%)$ than males $7 / 37(19 \%)$. OR diploma nurses were the majority
34/37(91.9\%). OR nurses with a working experience in the nursing field (10 years and more) were 30/37(81\%) and those who had working experience in OR (more than 10 years) were (70\%). OR nurses in general, cardiothoracic and orthopedic surgical specialty areas were $16 / 37(43 \%), 9 / 37(24 \%)$ and $12 / 37(33 \%)$ respectively.

\begin{tabular}{|c|c|c|}
\hline Background Data Related to OR Nurses' Variables & No. & $\%$ \\
\hline \multicolumn{3}{|l|}{ Infection Control Courses Attended by OR Nurses } \\
\hline Yes & 24 & $65 \%$ \\
\hline No & 13 & $35 \%$ \\
\hline \multicolumn{3}{|l|}{ Vaccination Status Against HBV } \\
\hline Yes & 30 & $81 \%$ \\
\hline No & 7 & $19 \%$ \\
\hline \multicolumn{3}{|l|}{ OR Nurses' Awareness Regarding Infection Control Ps \& Ps } \\
\hline Yes & 37 & $100 \%$ \\
\hline \multicolumn{3}{|l|}{ Number of Surgical Procedures Done in Each Specialty Area } \\
\hline General surgery & 40 & $42 \%$ \\
\hline Cardiothoracic surgery & 24 & $25 \%$ \\
\hline Orthopedic surgery & 32 & $33 \%$ \\
\hline \multicolumn{3}{|c|}{ Observation Hours of Surgical Procedures Done in the Three Specialty Areas (457 hrs) } \\
\hline$<3$ & 4 & $4.20 \%$ \\
\hline $3-$ & 80 & $83.30 \%$ \\
\hline 5- & 12 & $12.50 \%$ \\
\hline Mean \pm SD & \multicolumn{2}{|c|}{$4.76 \pm 0.72$} \\
\hline
\end{tabular}

Table 2 shows that OR nurses who attended IC courses were 24/37 (65\%), and who were vaccinated against HBV were 30/37 $(81 \%)$. All OR nurses in the three specialty areas were aware of IC policies and procedures. 40/96 (42\%) of the total surgical procedures were done in general surgery, while 32/96 (33\%) in orthopedic and 24/96 (25\%) in cardiothoracic surgery. Almost $(83.3 \%)$ of the total surgical procedures were continued for (three to less than five hours). The total observation hours of nurses' practices regarding SPs were 457 hours with a mean of $4.76 \pm 0.72$ hours.

Table 3 illustrates that the compliance level with headgear practice among OR nurses was 79/96 (82.3\%) (Moderate compliance level). Compliance level with the use of mask and surgical gloves were 62/96 (64.6\%) (Low compliance levels). The compliance with the use of OR boots, surgical hand washing, 
surgical gown, handling sharp instruments were 71/96 (74\%), 1/96 (1\%), 27/96 (28.1\%), and 30/96 (31.2\%) respectively (Low compliance levels). The compliance with goggles practice was zero, it was worth saying that, the total mean of compliance level with the eight SPs practices among OR nurses was low (43.22 \pm 32.54$)$.

\begin{tabular}{|c|c|c|c|}
\hline Table 3: Frequency and Percentage Distribution of Compliance Level with Each SP Practice $(\mathrm{N}=96)^{*}$. \\
\hline SP Practices & No. & $\%$ & Compliance level (Low-Moderate-High) \\
\hline Headgear & 79 & $82.30 \%$ & Moderate \\
\hline Surgical mask & 62 & $64.60 \%$ & Low \\
\hline Goggles & 0 & $0 \%$ & Low \\
\hline OR boots & 71 & $74 \%$ & Low \\
\hline Surgical hand washing & 1 & $28.10 \%$ & Low Low \\
\hline Surgical gown & 27 & $64.60 \%$ & Low \\
\hline Surgical gloves & 62 & $31.20 \%$ & Low \\
\hline Handling sharp instruments & 30 & & \\
\hline Mean \pm SD & $43.22 \pm 32.54$ & & \\
\hline
\end{tabular}

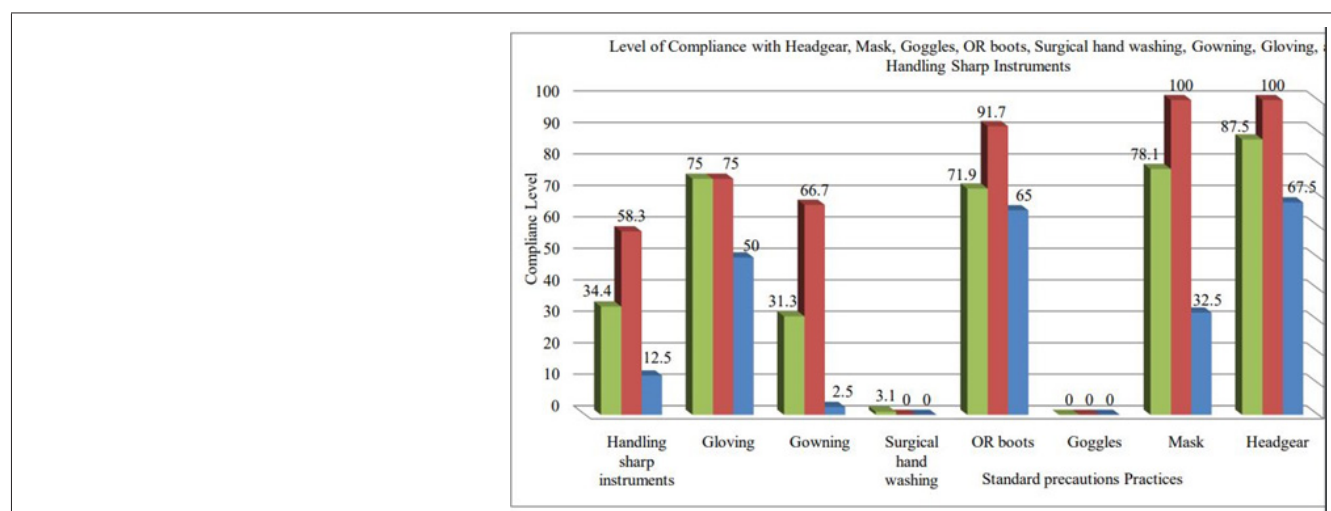

Figure 1: Highest level of compliance occurred in cardiothoracic surgery with the use of head gear and mask.

\begin{tabular}{|c|c|c|c|c|}
\hline Table 4: Frequency and Percentage Distribution of the Compliance Level with the Eight SPs Practices in Each Surgical Specialty areas. \\
\hline $\begin{array}{c}\text { OR Surgical Specialty } \\
\text { Areas }\end{array}$ & $\begin{array}{c}\text { Total Number of the Eight } \\
\text { SPs Practices (768) }\end{array}$ & No. & Compliance Level with the Eight SP Practices \\
\cline { 3 - 5 } & $40 \times 8=320$ & 92 & 28.75 & Low- Moderate- High) \\
\hline General & $24 \times 8=192$ & 118 & 41.46 & Low \\
\hline Cardiothoracic & $32 \times 8=256$ & 122 & 47.66 & Low \\
\hline Orthopedic & & & & \\
\hline
\end{tabular}

Table 4 shows that the compliance level with the eight SPs practices were low in the three surgical specialty areas; general 92/320 (28.75\%), cardiothoracic 118/192 (61.46\%), and orthopedic 122/256 (47.66\%). Figure 1 shows that the highest level of compliance occurred in cardiothoracic surgery with the use of head gear, and mask (100\%), (100\%) respectively. Compliance level with OR boots was moderate (91.7\%), While it was low with gowning, gloving, and handling sharp instruments and zero with goggles and surgical hand washing. In orthopedic surgery the level of compliance with headgear was moderate (87.5\%) and low with gowning, gloving, handling sharp instruments, surgical hand washing, and zero with goggles. The compliance level with SP practices in general surgery was the lowest in the three specialty areas. Headgear (67.5\%), mask (32.5\%), OR boots (65\%), gowning (2.5\%), gloving (50\%), handling sharp instruments $(12.5 \%)$, and zero with goggles and surgical hand washing.

\section{Discussion}

Although OR nurses in cardiothoracic specialty area had the highest compliance level with headgear and surgical mask practices, the total compliance level with the eight SPs was low. Cardiothoracic surgery area is the highest in risk regarding blood borne exposure as risk increases with longer, more invasive, and higher blood loss procedures. Consistent with other studies, in a survey study on compliance with SPs among OR nurses in South Korea, Jeong et al. [7] found that OR nurses rarely complied with SPs, especially with using protective eye wear (goggles). Also, in a study done by Ebied [8] she reported that masks, boots were rarely used by nurses when they dealt with blood or body fluids. Additionally, Abou El-Enein \& El Mahdy [9] mentioned that the compliance with protective eye wear (goggles) was zero and poor with surgical hand washing practice. On the contrary Cutter \& Jordan [10] showed that, half 
of the total sample of OR nurses in their study complied with eye protection for example.

\section{Conclusion}

In conclusion the compliance level with the SPs among OR nurses in this study was low. The results of this study are in agreement with those of previous studies that reported a less than $100 \%$ compliance rate with SPs, which has implications for staff safety, patient protection and the care environment. Furthermore, OR nurses were selective in their compliance to certain measures of the precautions.

\section{References}

1. Ganczak M, Szych Z (2007) Surgical nurses and compliance with personal protective equipment. J Hosp Infect 66(4): 346-351.

2. Spry C (2009) Essentials of perioperative nursing. ( $4^{\text {th }}$ edn), pp. 95-114.

3. Cullen BL, Genasi F, Symington I, Bagg J, Mc Creaddie M, et al. (2006) Potential for reported needlestick injury prevention among healthcare workers through safety device usage and improvement of guideline adherence: expert panel assessment. J Hosp Infect 63(4): 445-451.
4. Efstathiou G, Papastavrou E, Raftopoulos V, Merkouris A (2011) Compliance of Cypriot Nurses with Standard Precautions to avoid exposure to pathogens. Nurs Health Sci, 13(1): 53-59.

5. Centers for Disease Control and Prevention CDC (2005) Proceedings of the National Sharps Injury Prevention Meeting. Atlanta, GA, p. 1-26.

6. Centers for Disease Control and Prevention (1997) Evaluation of safety devices for preventing percutaneous injuries among healthcare workers during phlebotomy procedures Minneapolis St, Paul, New York City, and San Francisco. MMWR Morb Mortal Wkly Rep 46(2): 21-25.

7. Jeong I, Cho J, Park S (2008) Compliance with standard precautions among operating room nurses in South Korea. Am J Infect Control 36(10): 739-742.

8. Ebied E (2011) Impact of a Blood Borne Diseases Prevention Program on Compliance with Infection Control Standard Precaution Practices among Nurse in Family Health Centers, El Fayoum Governorate, Egypt.

9. Abou El Enein NY, El Mahdy HM (2010) Standard Precautions: A KAP Study among Nurses in the Dialysis Unit in A University Hospital in Alexandria, Egypt. J Egypt Public Health Assoc. 86(1-2): 3-10.

10. Cutter J, Jordan S (2012) Inter-professional differences in compliance with standard precautions in operating theatres: a multi-site, mixed methods study. Int J Nurs Stud 49(8):953-968. 